\title{
INEQUALITIES FOR SECTOR MATRICES AND POSITIVE LINEAR MAPS*
}

\author{
FUPING TAN $^{\dagger}$ AND HUIMIN CHEN ${ }^{\ddagger}$
}

Abstract. Ando proved that if $A, B$ are positive definite, then for any positive linear map $\Phi$, it holds

$$
\Phi\left(A \sharp_{\lambda} B\right) \leq \Phi(A) \sharp_{\lambda} \Phi(B),
$$

where $A \sharp_{\lambda} B, 0 \leq \lambda \leq 1$, means the weighted geometric mean of $A, B$. Using the recently defined geometric mean for accretive matrices, Ando's result is extended to sector matrices. Some norm inequalities are considered as well.

Key words. Positive linear maps, Geometric mean, Sector matrix, Norm inequality.

AMS subject classifications. 15A45, 15A60.

1. Introduction. Let $\mathbb{M}_{n}$ be the set of $n \times n$ complex matrices. Every $A \in \mathbb{M}_{n}$ could be decomposed as

$$
A=\Re A+i \Im A,
$$

where $A^{*}$ means the conjugate transpose of $A$ and $\Re A=\frac{A+A^{*}}{2}, \Im A=\frac{A-A^{*}}{2 i}$ are called the real, imaginary part of $A$, respectively. If $\Re A$ is positive definite, then we say $A$ is accretive. If both $\Re A$ and $\Im A$ are positive definite, then we say $A$ is accretive-dissipative. This class of matrices has received much attention over the past few years; see $[7,8,11,14,15,17,21]$ for example. For two Hermitian matrices $A, B \in \mathbb{M}_{n}$, we write $A \geq B$ (or $B \leq A)$ if $A-B$ is positive semidefinite. If $A, B \in \mathbb{M}_{n}$ are positive definite, the weighted geometric mean is defined as

$$
A \sharp_{\lambda} B=A^{1 / 2}\left(A^{-1 / 2} B A^{-1 / 2}\right)^{\lambda} A^{1 / 2} ;
$$

the weighted harmonic mean is defined as

$$
A !_{\lambda} B=\left((1-\lambda) A^{-1}+\lambda B\right)^{-1},
$$

where $0 \leq \lambda \leq 1$.

In 1979, Ando [1] proved that if $A, B \in \mathbb{M}_{n}$ are positive definite, then for any positive linear map $\Phi$, it holds

$$
\begin{aligned}
& \Phi\left(A \sharp_{\lambda} B\right) \leq \Phi(A) \sharp_{\lambda} \Phi(B), \\
& \Phi\left(A !_{\lambda} B\right) \leq \Phi(A) !_{\lambda} \Phi(B) .
\end{aligned}
$$

On the other hand, Choi's inequality (see e.g. [2, p. 41]) says that for any positive and unital linear map $\Phi$, it holds

$$
\Phi\left(A^{-1}\right) \geq \Phi(A)^{-1}
$$

${ }^{*}$ Received by the editors on May 7, 2019. Accepted for publication on August 9, 2019. Handling Editor: Ilya Spitkovsky. Corresponding Author: Fuping Tan.

${ }^{\dagger}$ Department of Mathematics, Shanghai University, Shanghai 200444, China (fptan@shu.edu.cn).

${ }_{\ddagger}^{\ddagger}$ Department of Mathematics, Shanghai University, Shanghai 200444, China (1670806425@qq.com). 
where $A \in \mathbb{M}_{n}$ is positive definite. For a survey on positive linear maps, we refer to Chapter 2 of [2].

In this article, we intend to extend (1.1), (1.2) and (1.3) to sector matrices. In the remaining part of this section, we introduce this class of matrices and the recently defined weighted geometric mean for accretive matrices. To our best knowledge, the connection between positive linear maps and sector matrices have not yet been explored. Our contribution in this article shows that such a connection would be a fruitful topic to investigate.

Define a sector $S_{\theta}$ on the complex plane

$$
S_{\theta}=\{z \in \mathbb{C}: \Re z>0,|\Im z| \leq(\Re z) \tan \theta\},
$$

where $\theta \in[0, \pi / 2)$ is fixed.

Recall that the numerical range (see, e.g., [9]) of $A \in \mathbb{M}_{n}$ is defined as the set on the complex plane

$$
W(A)=\left\{x^{*} A x: x \in \mathbb{C}^{n}, x^{*} x=1\right\} .
$$

In [12], if $W(A) \subset S_{\theta}$, then $A$ is called a sector matrix. Clearly, if $W(A) \subset S_{\theta}$, then $\Re A$ is positive definite. Therefore, a sector matrix is accretive with extra information about the specified angle $\theta$. Some recent studies of sector matrices can be found in $[4,5,6,12,19,20]$ and references therein.

The geometric mean of two accretive matrices $A, B \in \mathbb{M}_{n}$ was first brought in by Drury [4], who defined

$$
A \sharp B=\left(\frac{2}{\pi} \int_{0}^{\infty}\left(s A+s^{-1} B\right)^{-1} \frac{d s}{s}\right)^{-1},
$$

in which we continue to use the standard notation just as in the positive definite matrices case. Raissouli, Moslehian and Furuichi [18] recently defined the following weighted geometric mean of two accretive matrices $A, B \in \mathbb{M}_{n}$,

$$
A \sharp_{\lambda} B=\frac{\sin \lambda \pi}{\pi} \int_{0}^{\infty} s^{\lambda-1}\left(A^{-1}+s B^{-1}\right)^{-1} d s,
$$

where $\lambda \in[0,1]$. It could be verified that when $\lambda=1 / 2$, the formula (1.5) coincides with the formula (1.4).

The main results and their proofs are given in Section 3, after the preparation of technical lemmas in Section 2.

2. Lemmas. The first lemma gives the closure property of sector matrices under the positive linear map.

Lemma 1. Let $\Phi$ be a positive linear map. If $A \in \mathbb{M}_{n}$ with $W(A) \subset S_{\theta}$, then $W(\Phi(A)) \subset S_{\theta}$. In particular, if $A \in \mathbb{M}_{n}$ is accretive, then so is $\Phi(A)$.

Proof. First of all, note that for any $T \in \mathbb{M}_{n}$,

$$
\begin{aligned}
\Re \Phi(T) & =\left(\Phi(T)+\Phi(T)^{*}\right) / 2 \\
& =\left(\Phi(T)+\Phi\left(T^{*}\right)\right) / 2 \\
& =\Phi\left((T+T)^{*} / 2\right)=\Phi(\Re T),
\end{aligned}
$$

in which the second equality is by a lemma in $[2$, p. 50$]$. That is,

$$
\Re \Phi(T)=\Phi(\Re T) .
$$


Similarly, we have

$$
\Im \Phi(T)=\Phi(\Im T)
$$

Now since $W(A) \subset S_{\theta}$, by definition we have $\pm \Im A \leq(\tan \theta) \Re A$. Applying the map $\Phi$ to the previous inequality gives $\pm \Phi(\Im A) \leq(\tan \theta) \Phi(\Re A)$, equvalently, $\pm \Im \Phi(A) \leq(\tan \theta) \Re \Phi(A)$, that is, $W(\Phi(A)) \subset S_{\theta}$, as required.

Lemma 2. [13, Lemma 2.4] Let $A \in \mathbb{M}_{n}$ be accretive. Then

$$
(\Re A)^{-1} \geq \Re A^{-1} .
$$

A reverse of Lemma 2 is as follows.

Lemma 3. [12, Lemma 3] Let $A \in \mathbb{M}_{n}$ with $W(A) \subset S_{\theta}$. Then

$$
(\Re A)^{-1} \leq(\sec \theta)^{2} \Re A^{-1} .
$$

The following remarkable property about the weighted geometric mean of accretive matrices was proved by Raissouli, Moslehian and Furuichi.

Lemma 4. [18, Theorem 2.4] Let $A, B \in \mathbb{M}_{n}$ be accretive and let $\lambda \in[0,1]$. Then

$$
\Re\left(A \sharp_{\lambda} B\right) \geq(\Re A) \sharp_{\lambda}(\Re B) .
$$

We remark that when $\lambda=1 / 2$, Lemma 4 was observed in [16]. The next lemma complements Lemma 4.

Lemma 5. Let $A, B \in \mathbb{M}_{n}$ such that $W(A), W(B) \subset S_{\theta}$ and let $\lambda \in[0,1]$. Then

$$
(\cos \theta)^{2} \Re\left(A \sharp_{\lambda} B\right) \leq(\Re A) \sharp_{\lambda}(\Re B) .
$$

Proof. By Lemma 2, we have

$$
\Re\left(A^{-1}+\lambda B^{-1}\right)^{-1} \leq\left(\Re A^{-1}+\lambda \Re B^{-1}\right)^{-1} .
$$

On the other hand, by Lemma 3 we have

$$
\Re A^{-1}+\lambda \Re B^{-1} \geq(\cos \theta)^{2}\left((\Re A)^{-1}+\lambda(\Re B)^{-1}\right) .
$$

Thus,

$$
\Re\left(A^{-1}+\lambda B^{-1}\right)^{-1} \leq(\sec \theta)^{2}\left((\Re A)^{-1}+\lambda(\Re B)^{-1}\right)^{-1} .
$$

Combining previous two inequalities gives

$$
\begin{aligned}
\Re\left(A \sharp_{\lambda} B\right) & =\frac{\sin \lambda \pi}{\pi} \int_{0}^{\infty} \lambda^{t-1} \Re\left(A^{-1}+\lambda B^{-1}\right)^{-1} d t \\
& \leq \frac{\sin \lambda \pi}{\pi} \int_{0}^{\infty} \lambda^{t-1}(\sec \theta)^{2}\left((\Re A)^{-1}+\lambda(\Re B)^{-1}\right)^{-1} d t \\
& =(\sec \theta)^{2}\left((\Re A) \sharp_{\lambda}(\Re B)\right) .
\end{aligned}
$$

The desired result follows. 
Recall that a norm $\|\cdot\|$ on $\mathbb{M}_{n}$ is unitarily invariant if $\|U A V\|=\|A\|$ for any $A \in \mathbb{M}_{n}$ and for all unitary matrices $U, V \in \mathbb{M}_{n}$. The next lemma is known as the Fan-Hoffman inequality in the literature.

Lemma 6. [3, p. 74] Let $A \in \mathbb{M}_{n}$. Then for any unitarily invariant norm $\|\cdot\|$,

$$
\|\Re A\| \leq\|A\| .
$$

Lemma 7. [20] Let $A \in \mathbb{M}_{n}$ such that $W(A) \subset S_{\theta}$. Then for any unitarily invariant norm $\|\cdot\|$,

$$
\cos \theta\|A\| \leq\|\Re A\| .
$$

3. Main results. We start with an extension of (1.1).

Theorem 8. Let $A, B \in \mathbb{M}_{n}$ such that $W(A), W(B) \subset S_{\theta}$. Then for any positive linear map $\Phi$, it holds

$$
(\cos \theta)^{2} \Re \Phi\left(A \sharp_{\lambda} B\right) \leq \Re\left(\Phi(A) \sharp_{\lambda} \Phi(B)\right),
$$

where $\lambda \in[0,1]$.

Proof. Lemma 5 tells us that

$$
(\cos \theta)^{2} \Re\left(A \sharp_{\lambda} B\right) \leq(\Re A) \sharp_{\lambda}(\Re B) .
$$

Applying the positive linear map $\Phi$ to the previous inequality and by Lemma 1, we obtain

$$
(\cos \theta)^{2} \Re \Phi\left(A \sharp_{\lambda} B\right) \leq \Phi\left((\Re A) \sharp_{\lambda}(\Re B)\right) .
$$

Now we estimate

$$
\begin{aligned}
\Phi\left((\Re A) \sharp_{\lambda}(\Re B)\right) & \leq \Phi(\Re A) \sharp_{\lambda} \Phi(\Re B) \\
& =(\Re \Phi(A)) \sharp_{\lambda}(\Re \Phi(B)) \\
& \leq \Re\left(\Phi(A) \sharp_{\lambda} \Phi(B)\right),
\end{aligned}
$$

in which the first inequality by (1.1), the second inequality is by Lemma 4 .

Corollary 9. Let $A, B \in \mathbb{M}_{n}$ such that $W(A), W(B) \subset S_{\theta}$. Then for any positive linear map $\Phi$ and unitarily invariant norm $\|\cdot\|$, it holds

$$
(\cos \theta)^{3}\left\|\Phi\left(A \sharp_{\lambda} B\right)\right\| \leq\left\|\Phi(A) \sharp_{\lambda} \Phi(B)\right\|,
$$

where $\lambda \in[0,1]$.

Proof. By Lemma 7 and by Lemma 1, we have

$$
\cos \theta\left\|\Phi\left(A \sharp_{\lambda} B\right)\right\| \leq\left\|\Re \Phi\left(A \sharp_{\lambda} B\right)\right\| .
$$

Then by (3.7) and Lemma 6,

$$
(\cos \theta)^{2}\left\|\Re \Phi\left(A \sharp_{\lambda} B\right)\right\| \leq\left\|\Re\left(\Phi(A) \sharp_{\lambda} \Phi(B)\right)\right\| \leq\left\|\left(\Phi(A) \sharp_{\lambda} \Phi(B)\right)\right\| .
$$

The desired result follows. 
Corollary 10. Let $A, B \in \mathbb{M}_{n}$ be accretive-dissipative. Then for any positive linear map $\Phi$ and unitarily invariant norm $\|\cdot\|$, it holds

$$
\frac{\sqrt{2}}{4}\left\|\Phi\left(A \sharp_{\lambda} B\right)\right\| \leq\left\|\Phi(A) \sharp_{\lambda} \Phi(B)\right\|,
$$

where $\lambda \in[0,1]$.

Proof. It is easy to observe that $W\left(e^{-i \pi / 4} A\right) \subset S_{\pi / 4}, W\left(e^{-i \pi / 4} B\right) \subset S_{\pi / 4}$. Moreover, by (1.5),

$$
\begin{aligned}
\left(e^{-i \pi / 4} A\right) \sharp_{\lambda}\left(e^{-i \pi / 4} B\right) & =e^{-i \pi / 4} \frac{\sin \lambda \pi}{\pi} \int_{0}^{\infty} s^{\lambda-1}\left(A^{-1}+s B^{-1}\right)^{-1} d s \\
& =e^{-i \pi / 4}\left(A \sharp_{\lambda} B\right) .
\end{aligned}
$$

One readily finds that (3.9) follows from (3.8) by specifying $\theta$ to be equal to $\pi / 4$.

Using exactly the same approach, one could state analogous results for the weighted harmonic mean, we leave the details of the proof for the interested reader.

TheOREm 11. Let $A, B \in \mathbb{M}_{n}$ such that $W(A), W(B) \subset S_{\theta}$. Then for any positive linear map $\Phi$, it holds

$$
(\cos \theta)^{2} \Re \Phi\left(A !_{\lambda} B\right) \leq \Re\left(\Phi(A) !_{\lambda} \Phi(B)\right),
$$

where $\lambda \in[0,1]$.

Corollary 12. Let $A, B \in \mathbb{M}_{n}$ such that $W(A), W(B) \subset S_{\theta}$. Then for any positive linear map $\Phi$ and unitarily invariant norm $\|\cdot\|$, it holds

$$
(\cos \theta)^{3}\left\|\Phi\left(A !_{\lambda} B\right)\right\| \leq\left\|\Phi(A) !_{\lambda} \Phi(B)\right\|,
$$

where $\lambda \in[0,1]$.

Corollary 13. Let $A, B \in \mathbb{M}_{n}$ be accretive-dissipative. Then for any positive linear map $\Phi$ and unitarily invariant norm $\|\cdot\|$, it holds

$$
\frac{\sqrt{2}}{4}\left\|\Phi\left(A !_{\lambda} B\right)\right\| \leq\left\|\Phi(A) !_{\lambda} \Phi(B)\right\|,
$$

where $\lambda \in[0,1]$.

Next, we present an extension of (1.3) and related norm inequalities.

Theorem 14. Let $A \in \mathbb{M}_{n}$ such that $W(A) \subset S_{\theta}$. Then for any positive unital linear map $\Phi$, it holds

$$
(\cos \theta)^{2} \Re \Phi^{-1}(A) \leq \Re \Phi\left(A^{-1}\right) .
$$

Proof. By Lemma 1 and Lemma 2, we have

$$
\Re \Phi^{-1}(A) \leq(\Re \Phi(A))^{-1}=(\Phi(\Re A))^{-1} .
$$

Now by Choi's inequality (1.3), $(\Phi(\Re A))^{-1} \leq \Phi\left((\Re A)^{-1}\right)$. Finally, by Lemma 3, we have $\Phi\left((\Re A)^{-1}\right) \leq$ $(\sec \theta)^{2} \Phi\left(\Re A^{-1}\right)=(\sec \theta)^{2} \Re \Phi\left(A^{-1}\right)$. So the desired result follows. 
Similar to the proof of Corollary 9 and Corollary 10, we could present the following results.

Corollary 15. Let $A \in \mathbb{M}_{n}$ such that $W(A) \subset S_{\theta}$. Then for any positive unital linear map $\Phi$ and unitarily invariant norm $\|\cdot\|$, it holds

$$
(\cos \theta)^{3}\left\|\Phi^{-1}(A)\right\| \leq\left\|\Phi\left(A^{-1}\right)\right\|
$$

Corollary 16. Let $A \in \mathbb{M}_{n}$ be accretive-dissipative. Then for any positive unital linear map $\Phi$ and unitarily invariant norm $\|\cdot\|$, it holds

$$
\frac{\sqrt{2}}{4}\left\|\Phi^{-1}(A)\right\| \leq\left\|\Phi\left(A^{-1}\right)\right\|
$$

Acknowledgment. This work was supported by the National Nature Science Foundation of China (grant no. 11771275).

\section{REFERENCES}

[1] T. Ando. Concavity of certain maps on positive definite matrices and applications to Hadamard products. Linear Algebra Appl., 26:203-241, 1979.

[2] R. Bhatia. Positive Definite Matrices. Princeton University Press, Princeton, 2007.

[3] R. Bhatia. Matrix Analysis. Graduate Texts in Mathematics, Vol. 169, Springer-Verlag, New York, 1997.

[4] S. Drury. Principal powers of matrices with positive definite real part. Linear Multilinear Algebra, 63:296-301, 2015.

[5] S. Drury and M. Lin. Singular value inequalities for matrices with numerical ranges in a sector. Oper. Matrices, 8:1143$1148,2014$.

[6] X. Fu and Y. Liu. Rotfeld inequality for partitioned matrices with numerical ranges in a sector. Linear Multilinear Algebra, 64:105-109, 2016.

[7] A. George, Kh.D. Ikramov, and A.B. Kucherov. On the growth factor in Gaussian elimination for generalized Higham matrices. Numer. Linear Algebra Appl., 9:107-114, 2002.

[8] I. H. Gumus, O. Hirzallah, and F. Kittaneh. Norm inequalities involving accretive-dissipative $2 \times 2$ block matrices. Linear Algebra Appl., 528:76-93, 2017.

[9] K.E. Gustafson and D.K.M. Rao. Numerical Range: The Field of Values of Linear Operators and Matrices. Springer, New York, 1997.

[10] R.A. Horn and C.R. Johnson. Matrix Analysis. Cambridge University Press, Cambridge, 2013.

[11] F. Kittaneh and M. Sakkijha. Inequalities for accretive-dissipative matrices. Linear Multilinear Algebra, 67:1037-1042, 2019.

[12] M. Lin. Some inequalities for sector matrices. Oper. Matrices, 10:915-921, 2016.

[13] M. Lin. Extension of a result of Hanynsworth and Hartfiel. Arch. Math., 104(1):93-100, 2015.

[14] M. Lin. A note on the growth factor in Gaussian elimination for accretive-dissipative matrices. Calcolo, 51:363-366, 2014.

[15] M. Lin. Fischer type determinantal inequalities for accretive-dissipative matrices. Linear Algebra Appl., 438:2808-2812, 2013.

[16] M. Lin and F. Sun. A property of the geometric mean of accretive operator. Linear Multilinear Algebra, 65:433-437, 2017.

[17] M. Lin and D. Zhou. Norm inequalities for accretive-dissipative operator matrices. J. Math. Anal. Appl., 407:436-442, 2013.

[18] M. Raissouli, M.S. Moslehian, and S. Furuichi. Relative entropy and Tsallis entropy of two accretive operators. $C . R$. Math. Acad. Sci. Paris, Ser. I, 355:687-693, 2017.

[19] C. Yang and F. Lu. Some generalizations of inequalities for sector matrices. J. Inequal. Appl., 2018:Article no. 183, 2018

[20] F. Zhang. A matrix decomposition and its applications. Linear Multilinear Algebra, 63:2033-2042, 2015.

[21] Y. Zhang. Unitarily invariant norm inequalities for accretive-dissipative operators. J. Math. Anal. Appl., 412:564-569, 2014. 